\title{
Improving Continuing Education for Family Physicians: The Role of the American Board of Family Medicine
}

\author{
Warren P. Newton, MD, MPH, Elizabeth Baxley, MD, Kevin Rode, BA, \\ Tom O'Neill, PhD, and Roger Fain, $M A$
}

Continuing medical education (CME) is ground zero for improvement of health care. Although the last decade has seen great innovation in health professions education, ${ }^{1-3}$ only a small percentage of physicians are enrolled in any given time in formal, structured medical education. ${ }^{4}$ If care is going to improve, if innovation is going to spread, and if America wants changes in health care to occur more rapidly than the 30 years it takes to replace generations of health professionals, change must spread through a comprehensive and meaningful approach to lifelong learning. Therefore, as a specialty and as a profession, we must attend closely to the vitality and evolution of continuing medical education.

What do we know about the effectiveness of our continuing medical education system? Contemporary CME in the United States is a vast system, with 180,000 courses offered by 1750 accredited organizations in 2018, comprising more than 1.2 million hours of instruction with registration fees totaling $\$ 1,527,559,796 .^{5}$ Since 2017 , the number of educational events has increased $10 \%$ and hours of instruction increased by $5 \%$. As underscored in numerous evidence-based reviews, ${ }^{6-8}$ there is good evidence that the right kind of continuing education can increase knowledge and improve clinical performance. In particular, there is an emerging consensus that educational approaches that engage learners, use multiple modalities, and use spaced repetition can change clinical behavior and improve clinical outcomes. Unfortunately, the majority of continuing education in the United States remains relatively passive and single point in time $e^{5,9}$ and therefore less likely to be effective in impacting clinical outcomes in a meaningful way.

Conflict of interest: The authors are employees of the ABFM.
What about family medicine? A proud moment in the history of our specialty was the commitment made by the American Academy of General Practice (AAGP) in 1947 to require 50 hours of CME annually. Our founders made this commitment to acknowledge the importance and difficulty of keeping up to date as generalists. The AAGP changed its name to the American Academy of Family Practice (AAFP) in 1970; the AAFP and its chapters have continued to make CME a priority. The American Board of Family Medicine (ABFM) incorporated the AAFP requirement into its own requirements for board certification at its founding 50 years ago. Because of our collective commitment to the need for cognitive expertise and the recognition that a generalist specialty requires rigor, requirements for continuing education for board certification of family physicians are the highest of any specialty.

What role should the American Board of Family Medicine play in the larger continuing medical education system for family physicians? We believe that the primary responsibility for delivery of continuing education should be with the AAFP, its chapters and other partners interested in improving care. The most important contributions of the $\mathrm{ABFM}$, in addition to maintaining the longstanding CME requirement are in the provision of ways for physicians to have periodic, independent assessment of cognitive expertise over time- that is, the examination. There is ample evidence that physicians, like other professionals, are not able assess their own knowledge accurately: we do not know our clinical blind spots. ${ }^{10-13}$ For this reason, a keeping up-to-date strategy emphasizing self-selected CME by itself is incomplete. External assessment of knowledge, conducted in an objective and scientifically rigorous manner, and free of influ- 
ences of pharmaceutical companies, device makers, and other health care vendors is critical. This is also what the public expects from certifying boards.

The right amount of time between external assessments is a matter of judgment; practical aspects of cost and convenience are part of this consideration. At its founding, ABFM required reassessment of cognitive expertise every 7 years. In the early 2000s, as we evolved into a maintenance of certification model with requirements to participate in knowledge self-assessments (KSAs) and performance improvement activities every 3 years, we extended the maximal time between examinations to every 10 years. In addition to an overall pass/fail assessment, which compares an individual's cognitive expertise to a common standard applied to all Diplomates, ABFM also provides examinees a report highlighting strengths and areas in need of improvement, according to an examination blueprint based on organ systems. Importantly, the statistical precision of these estimates of knowledge gaps is only modest, as the ABFM examination is powered for the assessment of overall cognitive expertise. For example, the typical examination has only 3 questions related to the male reproductive system, based on the relative prevalence of these types of problems in typical family physicians' clinical practice. If a Diplomate misses all 3 questions, it may be appropriate to address this perceived gap with CME, but the small number of questions, as compared with the vast number of conditions affecting the male reproductive system, cannot provide conclusive evidence that his or her knowledge of such conditions is significantly worse than the average Diplomate.

The Family Medicine Certification Longitudinal Assessment (FMCLA) pilot has the potential to enhance Diplomates' efforts to remain current in their clinical knowledge. ${ }^{14}$ Pilot participants immediately receive the correct answer to each question, along with explanations for correct and incorrect answers in a summary critique and references that can be used for further study. Participants will receive interim reports with an estimated scaled score after they complete 100 questions. These reports will give Diplomates the opportunity to forecast whether their knowledge level is likely to be at or above passing the examination's minimum passing standard. The report will also include an individualized list on knowledge gaps linked to organ systems similar to that currently provided after the traditional point-in-time, 1-day examination. Our hope is that immediate and continuous feedback will help Diplomates develop a personalized learning plan, and that the ultimate result is realized in improvements in the quality of care they provide. The Continuous Knowledge Self Assessment (CKSA) will provide similar feedback, and continues to be a good of preparing for FMCLA.

ABFM's task now is to support better the efforts of family physicians in their lifelong learning between examinations. A first step will be to enhance the reports given to individual Diplomates at the 100-question mark. In early 2020, we will begin to identify more specifically the clinical topics missed and relate those to the Diplomate's self-rated relevance to their practice and confidence. Thus, a report might include clinical topics that Diplomate missed, but thought were relevant to his/her practice or the clinician was confident that he/she knew the answer. Our hope is that this kind of report will allow Diplomates to prioritize their learning needs and plan their continuing education.

In the midterm future, we will be validating a new examination blueprint organized around the age of the patient and the urgency and duration of the condition. For example, a sprained ankle is an acute condition that is self-limited, whereas hypertension is usually not urgent but will probably require lifelong management. These 2 conditions require different types of care and different types of knowledge. Initial reviews by experienced clinicians suggest that this new blueprint better captures how family physicians think about the clinical problems they face. We believe that the new blueprint, once validated, will be more useful to Diplomates' efforts to identify knowledge gaps. We have also begun to explore with the AAFP better alignment of taxonomy of topics and knowledge gaps, in hopes that Diplomates can better identify what they need to know and our education partners can better develop educational programs.

KSAs will remain an important part of our strategy. Currently KSAs are very popular among Diplomates, with approximately 3 times as many Diplomates doing them as would be required in any given year. We will continue to update KSAs annually; in addition, this year we will be reviewing the list of topics and begin to add new topics addressing burning public health issues (such as opioid use disorders), population health (such as multimorbidity) or support broad scope of practice. Regardless of topic, our intent is to provide addi- 
tional opportunities for self assessment-and to provide Diplomates an opportunity to practice their skills in self assessment.

Finally, following the lead of the American Board of Obstetrics and Gynecology and other American Board of Medical Specialties (ABMS) Boards, we will be developing a pilot of an optional national Family Medicine Journal Club, which will provide practice changing articles selected for relevance and methodological rigor from 140 clinical journals. Participating physicians will answer questions for each article selected to demonstrate understanding of the clinical application of the information and receive certification credit. As with our current self-assessment modules, we anticipate that this offering will provide opportunities for the AAFP, its chapters, or other interested CME providers to develop continuing education opportunities to meet the needs of our Diplomates.

Our work to improve the effectiveness of CME will not stop there. ABFM is acutely aware of the dramatic changes taking place in continuing education and, especially, in technology. We stand ready to work with Diplomates, the AAFP, and our other educational partners to optimize how family physicians maintain the cognitive expertise to improve care continuously. With new methods of providing continuing education will come new challenges, including the need to evaluate their effectiveness. Is novel necessarily better? Beyond this, additional scrutiny will be needed with respect to potentially new kinds of conflicts of interest posed by health system consolidation, integration of health systems with payers and the role of venture capital in clinical technology startups.

ABFM's long-term focus continues to be continuous improvement in the quality of care family physicians provide. Given that the continuing education of family physicians must play a central role in this process, the American Board of Family Medicine is committed to supporting its partners' efforts to improve the effectiveness of CME in improving clinical, outcomes.
To see this article online, please go to: http://jabfm.org/content/ 32/5/756.full.

\section{References}

1. Accelerating change in medical education. Available from: https://www.ama-assn.org/education/acceleratingchange-medical-education. Accessed July 26, 2019.

2. Our work in competency based medical education. Available from: https://www.aamc.org/initiatives/ meded/498322/ourworkoncompetencybasedmedical education.html. Accessed July 26, 2019.

3. Bacon TJ, Newton WP. Innovations in the education of health professionals. N C Med J 2014;75: $22-7$.

4. Fraher E, Ricketts TC. Building a Value-Based Workforce in North Carolina. North Carolina Medical Journal. 2016;77:94-8.

5. ACCME Data Report: Growth and advancement in accredited continuing medical education-2018. Available from: http://www.accme.org/publications/ accme-2018-data-report. Accessed July 26, 2019.

6. Cervero RM, Gaines JK. The impact of CME on physician performance and patient health outcomes: an updated synthesis of systematic reviews. J Contin Educ Health Prof 2015;35:131-8.

7. Bloom BS. Effects of continuing medical education on improving physician clinical care and patient health: a review of systematic reviews. Int $\mathrm{J}$ Technol Assess Health Care 2005;21:380-5.

8. Mansouri M, Lockyer J. A meta-analysis of continuing medical education effectiveness. J Contin Educ Health Prof 2007;27:6-15.

9. McMahon GT. What do I need to learn today?-the Evolution of CME. N Engl J Med 2016;374:1403-6.

10. Gordon MJ. A review of the validity and accuracy of self-assessments in health professions training. Acad Med 1991;66:762-9.

11. Gordon MJ. Self-assessment programs and their implications for health professions training. Acad Med 1992;67:672-9.

12. Davis DA, Mazmanian PE, Fordis M, Van Harrison R, Thorpe KE, Perrier L. Accuracy of physician self-assessment compared with observed measures of competence: a systematic review. JAMA 2006;296: 1094-102.

13. Eva KW, Regehr G. Self-assessment in the health professions: a reformulation and research agenda. Acad Med 2005;80(10)(Suppl):S46-S54.

14. Newton WP, Rode K, Fain R, Baxley E. Longitudinal assessment: Where we are and why it is important. J Am Board Fam Med 2019;32:448-50. 